Introduction and objectives The clinical course in patients with IPF is characterised by substantial inter- and intra-subject variability in the rates of disease progression, thereby confounding clinical assessments of therapeutic responses in individual patients. We pooled data from three Phase 3 trials to assess the potential benefit of continued treatment with pirfenidone in patients who experienced a $\geq 10 \%$ decline in percent predicted forced vital capacity $(\% \mathrm{FVC})$ during the first 6 months of treatment.

Methods Source data included all patients randomised to treatment with pirfenidone $2403 \mathrm{mg} / \mathrm{d}$ or placebo in the Phase 3 ASCEND or CAPACITY studies $(\mathrm{N}=1247)$. We selected patients with a $\geq 10 \%$ absolute decline in $\% \mathrm{FVC}$ by the month 3 or 6 study visit and compared the proportion of patients in the pirfenidone and placebo groups who experienced any of the following during the subsequent 6-month interval: (1) $\geq 10 \%$ absolute decline in $\% \mathrm{FVC}$ or death; (2) no further decline in $\% \mathrm{FVC}$; or (3) death. Observed data were used in the analysis.

Results 34 (5.5\%) and 68 (10.9\%) patients in the pooled pirfenidone and placebo groups, respectively, experienced a $\geq 10 \%$ absolute decline in $\%$ FVC between baseline and month 6 (relative difference, 49.5\%). Analysis of outcomes during the subsequent 6-month interval demonstrated that fewer patients in the pirfenidone group compared with placebo experienced a $\geq 10 \%$ decline in $\%$ FVC or death (pirfenidone, 2/34 [5.9\%] vs. placebo, 19/68 [27.9\%]). More patients in the pirfenidone group compared with placebo had no further decline in\%FVC (20/34 [58.8\%] vs. 26/68 [38.2\%]; Table 1). Additionally, there were fewer deaths in the pirfenidone group (1/34 [2.9\%]) compared with placebo (14/68 [20.6\%]).

\begin{tabular}{|c|c|c|c|c|}
\hline Outcome, n (\%) & $\begin{array}{l}\text { Pirfenidone } \\
(\mathrm{n}=34)\end{array}$ & $\begin{array}{l}\text { Placebo } \\
(\mathrm{n}=68)\end{array}$ & Relative difference* & $P$ value ${ }^{\dagger}$ \\
\hline $\begin{array}{l}\geq 10 \% \text { decline in FVC } \\
\text { or death }\end{array}$ & $2(5.9)$ & $19(27.9)$ & $-78.9 \%$ & 0.009 \\
\hline Death & $1(2.9)$ & $14(20.6)$ & $-85.7 \%$ & 0.018 \\
\hline$>0 \%$ to $<10 \%$ decline in FVC & $12(35.3)$ & $23(33.8)$ & $4.3 \%$ & ND \\
\hline No further decline in $\mathrm{FVC}^{\ddagger}$ & $20(58.8)$ & $26(38.2)$ & $53.8 \%$ & 0.059 \\
\hline
\end{tabular}

Conclusions Among patients who experienced a $\geq 10 \%$ decline in\%FVC during the first 6 months of treatment, continued treatment with pirfenidone resulted in a lower risk of $\% \mathrm{FVC}$ decline or death during the subsequent 6 months. These findings suggest a potential benefit to continued treatment with pirfenidone despite an initial decline in FVC.

\section{S110 EFFICACY AND SAFETY OF NINTEDANIB IN PATIENTS WITH IPF BEYOND WEEK 52: DATA FROM THE PHASE II TOMORROW TRIAL}

${ }^{1} \mathrm{~L}$ Richeldi, ${ }^{2} \mathrm{U}$ Costabel, ${ }^{3} \mathrm{M}$ Selman, ${ }^{4} \mathrm{Z} \mathrm{Xu},{ }^{5} \mathrm{~T}$ Kimura, ${ }^{5} \mathrm{~S}$ Stowasser, ${ }^{5} \mathrm{C}$ Hallmann. ${ }^{1}$ National Institute for Health Research Southampton Respiratory Biomedical Research Unit and Clinical and Experimental Sciences, University of Southampton, Southampton, UK; ${ }^{2}$ Ruhrlandklinik, University Hospital, University of Duisburg-Essen, Essen, Germany; ${ }^{3}$ Instituto Nacional de Enfermedades Respiratorias, Mexico City, Mexico; ${ }^{4}$ Peking Union Medical College Hospital, Beijing, China; ${ }^{5}$ Boehringer Ingelheim Pharma GmbH \& Co. KG, Ingelheim Am Rhein, Germany

\subsection{6/thoraxjn-2015-207770.116}

Introduction Period 1 of the TOMORROW trial was a phase II, randomised, placebo-controlled trial of four doses of nintedanib (50 mg qd, $50 \mathrm{mg}$ bid, $100 \mathrm{mg}$ bid, $150 \mathrm{mg} \mathrm{bid}$ ) in patients with idiopathic pulmonary fibrosis (IPF) over 52 weeks. Results suggested that nintedanib $150 \mathrm{mg}$ bid was associated with a reduced decline in forced vital capacity (FVC) and fewer acute exacerbations versus placebo. After completing period 1, patients could continue treatment in a further blinded treatment phase (period 2).

Methods In period 2, patients treated with nintedanib in period 1 continued their dose, and placebo-treated patients switched to nintedanib $50 \mathrm{mg}$ qd. Here we present descriptive data on FVC, acute exacerbations, mortality and adverse events for the nintedanib $150 \mathrm{mg}$ bid and comparator (placebo/nintedanib $50 \mathrm{mg}$ qd) groups during periods 1 and 2 .

Results Of 428 patients treated in period 1 (nintedanib $150 \mathrm{mg}$ bid $\mathrm{n}=85$, placebo $\mathrm{n}=85$ ), 316 patients completed period 1 and 286 patients (nintedanib $150 \mathrm{mg}$ bid $\mathrm{n}=48$, comparator $\mathrm{n}$ $=54$ ) continued treatment in period 2. Mean total duration of exposure was 14.2 months for nintedanib $150 \mathrm{mg}$ bid and 16.8 months for comparator. The mean observed change from baseline in FVC over time was consistently lower in the nintedanib $150 \mathrm{mg}$ bid group compared with comparator across periods 1 and 2 (Figure 1). The incidence of acute exacerbations was lower in the nintedanib $150 \mathrm{mg}$ bid group compared with comparator (3.2 vs. 13.4 per 100 patient-years). Overall, 14 patients $(16.3 \%)$ died in the nintedanib $150 \mathrm{mg}$ bid group and 19 patients $(21.8 \%)$ died in the comparator group. The safety and tolerability of nintedanib $150 \mathrm{mg}$ bid was similar between periods 1 and 2. The proportion of patients reporting an adverse event across periods 1 and 2 was similar in the nintedanib 150 $\mathrm{mg}$ bid and comparator groups $\mathbf{( 9 7 . 6 \%}$ and $94.1 \%$, respectively).

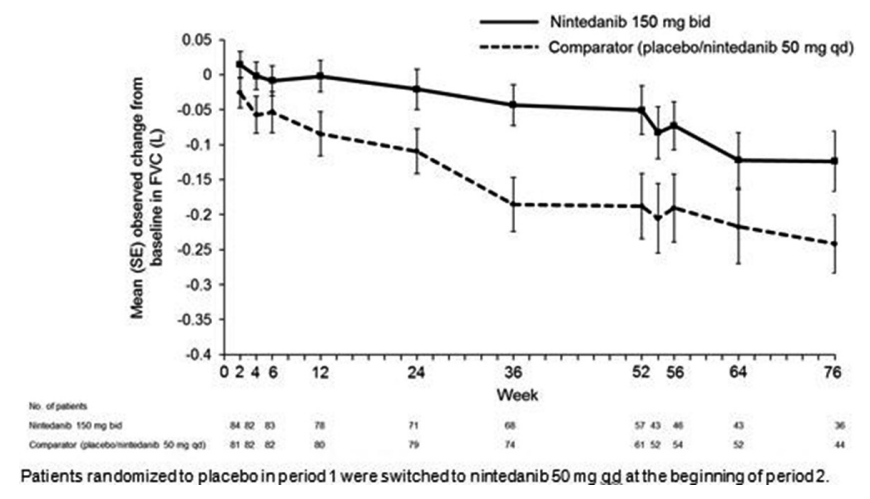

Abstract S110 Figure 1 
Conclusion In the TOMORROW trial, the effect of nintedanib on slowing disease progression in patients with IPF was maintained up to week 76. No relevant changes in the safety and tolerability of nintedanib were observed with treatment up to week 76 compared with week 52 .

\section{S111 DOES RATE OF DECLINE IN LUNG FUNCTION PREDICT RESPONSE TO PIRFENIDONE THERAPY IN PATIENTS WITH IDIOPATHIC PULMONARY FIBROSIS?}

JA Eaden, C Barber, SM Bianchi. Academic Directorate of Respiratory Medicine, Sheffield Teaching Hospitals NHS Foundation Trust, Sheffield, UK

\subsection{6/thoraxjnl-2015-207770.117}

Introduction and objectives Idiopathic pulmonary fibrosis (IPF) is a progressive, irreversible interstitial lung disease with a poor prognosis. Randomised, placebo-controlled clinical trials have shown that treatment of IPF patients with pirfenidone reduces disease progression. Published studies used incident forced vital capacity (FVC) values for study inclusion. Our objective was to determine whether the rate of decline in lung function predicts response to therapy.

Methods The clinical records of 68 patients with IPF who started pirfenidone treatment between June 2013 and March 2015 at a UK tertiary referral centre were reviewed. 34 patients had lung function sufficient for a full evaluation.

Results The mean (+/-SE) rate of decline in FVC per year pretreatment was $196.0+/-66.1 \mathrm{ml}$ versus $135.0+/-61.8 \mathrm{ml}$ post treatment $(\mathrm{p}=0.512)$. The decline in percent predicted FVC per year pre-treatment was $4.4+/-1.9 \%$ vs. $3.0+/-1.9 \%$ post treatment $(\mathrm{p}=0.572)$. The decline in absolute total gas transfer (TLco) per year pre-treatment was $0.6+/-0.2$ compared to 0.4 $+/-0.2$ post treatment $(\mathrm{p}=0.472)$. The decline in percent predicted TLco per year pre-treatment was $6.7+/-2.8 \%$ compared to $4.4+/-2.0 \%$ post treatment $(\mathrm{p}=0.504)$. Pirfenidone showed a trend towards positive benefit in all parameters measured. Patients were stratified into tertiles of slow, medium and rapid rate of FVC decline (pre-treatment). In slow and medium rate decliners (up to FVC decline of approximately $200 \mathrm{ml}$ per year or $5.4 \%$ per year) no effect was seen $(\mathrm{p}=\mathrm{NS})$. In fast decliners (FVC decline $576.1+/-97.8 \mathrm{ml}$ and $\mathrm{FVC}$ percent predicted decline $14.5+/-2.4 \%$ per year) a statistically significant effect was seen, reducing decline to $199.1+/-99.6 \mathrm{ml}$ and $4.5+/-2.4 \%$ respectively $(\mathrm{p}=0.022 ; 0.015)$.

$16.2 \%$ of the patients died with a mean survival of 239 days post pirfenidone.

Rate of decline in \% predicted FVC pre and post Pirfenidone stratified by rate of lung function decline pre-treatment

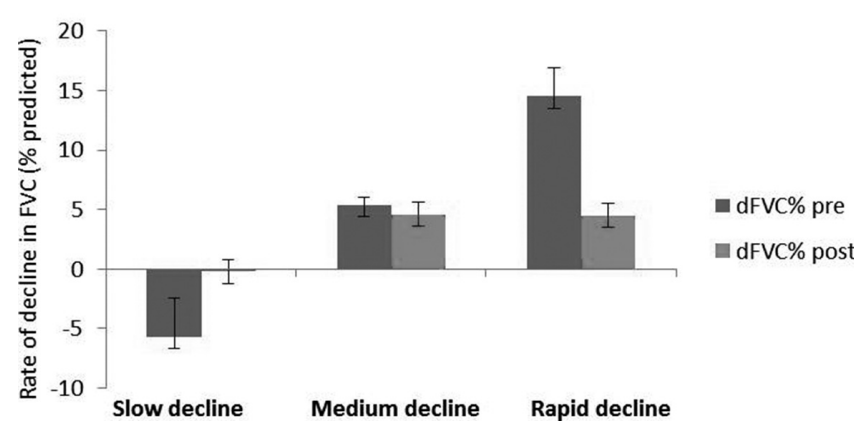

Abstract S111 Figure 1
Conclusions Pirfenidone treatment reduced disease progression (decline in FVC and TLCo) in our total cohort. Our results indicate that patients with rapid decline in FVC may benefit most from pirfenidone. Those declining at less than $200 \mathrm{ml}$ per year may not benefit. These results suggest that further clinical studies are warranted, including patients with evidence of rapid lung function decline with FVC $>80 \%$ predicted.

\section{Pseudomonas: digging for gold or search and destroy?}

\section{S112 VARIABILITY IN SUSCEPTIBILITY TO ANTIBIOTICS AND BACTERIOPHAGES BETWEEN INDIVIDUAL COLONIES OF PSEUDOMONAS AERUGINOSA FROM CYSTIC FIBROSIS SPUTUM SAMPLES: IMPLICATIONS FOR FUTURE CLINICAL TRIAL DESIGN}

${ }^{1} \mathrm{~V}$ Khoo, ${ }^{1} \mathrm{R}$ Pabary, ${ }^{1} \mathrm{H}$ Lund Palau, ${ }^{1} \mathrm{~A}$ Turnbull, ${ }^{2} \mathrm{~N}$ Madden, ${ }^{2} \mathrm{~S}$ Schelenz, ${ }^{2} \mathrm{~A}$ Jones, ${ }^{2} \mathrm{~S}$ Morales, ${ }^{1}$ EWFW Alton, ${ }^{1} \mathrm{JC}$ Davies. 'Imperial College, London, UK; ${ }^{2}$ Royal Brompton \& Harefield NHS Foundation Trust, London, UK

\subsection{6/thoraxjnl-2015-207770.118}

Antimicrobial resistance is a growing problem. As part of the Cystic Fibrosis (CF) Trust-funded Strategic Research Centre for research into Pseudomonas aeruginosa (Pa) (http://www.cysticfibrosis.org.uk/research-care/research/about-cystic-fibrosis-research/ how-we-invest-in-research/strategic-research-centres/src-1), we are exploring the therapeutic potential of bacteriophages. To date, we have a) identified a mix of 4 bacteriophages to which $>80 \%$ of our CF isolates appear sensitive; b) confirmed efficacy in an acute murine model and c) confirmed compatibility with clinically relevant nebulisers. In the clinical trial, patients will be selected on the basis of confirmed sensitivity of their Pa strain (s) to the bacteriophage mix. Significant variability has previously been reported when multiple, morphologically indistinguishable colonies were tested against antibiotics; here, we explored whether a similar phenomenon occurs with bacteriophages.

CF sputum ( $\mathrm{n}=6$ patients) was cultured on Pa-specific agar. Up to 10 individual morphologically identical colonies were inoculated separately into broth. After overnight culture each was subjected to disc diffusion (12 antibiotics) and phage testing (standard plaque assays).

Reproducibility (repeat testing of same broth) was excellent for susceptibility/resistance to antibiotics $(97.2 \%$ within $3 \mathrm{~mm})$ and bacteriophages (93.3\% within one log dilution). Variability in antibiotic susceptibility was lower than anticipated: in no samples were there both completely resistant and fully sensitive colonies and although zone sizes did vary, these crossed break points in only $12.5 \%$ of assessable samples. There was more variability in phage sensitivity. Colonies from two subjects displayed $<1 \mathrm{log}$ dilution differences between them and were therefore reasonably consistent. The other 4 subjects displayed $>1 \log$ differences between the most and least sensitive; in one, colonies clearly fell into two groups (highly sensitive/less so with a $4 \log$ dilution difference) and on subculture, differences in pigment production were apparent. Susceptibility may not be predicted by testing a mix of colonies.

As described previously for antibiotics, individual, apparently morphologically similar colonies of $\mathrm{Pa}$ in a CF sputum sample display inconsistent susceptibility profiles to anti-pseudomonal bacteriophages. The potential role of genetic variability to 